SI $95-16$

February 1995

\title{
Hard diffraction in hadron-hadron interactions and in photoproduction
}

\author{
R. Engel ${ }^{*, \dagger}$, J. Ranft ${ }^{\ddagger}$, S. Roesler ${ }^{\dagger}$ \\ *Universität Leipzig, Fachbereich Physik, D-04109 Leipzig, Germany \\ ${ }^{\dagger}$ Universität Siegen, Fachbereich Physik, D-57068 Siegen, Germany \\ ${ }^{\ddagger}$ CERN, CH-1211 Genève 23, Switzerland
}

\begin{abstract}
Hard single diffractive processes are studied within the framework of the triple-Pomeron approximation. Using a Pomeron structure function motivated by Regge-theory we obtain parton distribution functions which do not obey momentum sum rule. Based on Regge- factorization cross sections for hard diffraction are calculated. Furthermore, the model is applied to hard diffractive particle production in photoproduction and in $p \bar{p}$ interactions.
\end{abstract}




\section{Introduction}

A significant fraction of events in high energy hadron-hadron interactions and in photoproduction is characterized by a rapidity gap between a quasi-elastically scattered primary hadron and a multiparticle final state. These single diffractive interactions can be understood in terms of Pomeron exchange.

As suggested by Ingelman and Schlein [1] there is now - in addition to the well-investigated soft diffractive particle production - experimental evidence for single diffractive jet production both in $p \bar{p}$ and ep interactions [2, 3, 4, 5, 6, 7]. Characteristic features of these jets are very similar to the ones produced in nondiffractive events. This observation suggests that it might be possible to apply models based on perturbative QCD to describe hard diffractive scattering processes between hadrons or photons and Pomerons.

It is useful to distinguish between noncoherent diffraction and processes where the Pomeron enters the hard scattering as a whole. Models based on noncoherent interactions usually assume the Pomeron to be a partonic object, the partonmomenta obeying parton distribution functions (PDFs) [1, 8, 9, 10, 11, 12]. According to the assumptions made, these PDFs strongly differ in the partons considered, in their shapes, and in their normalizations. In addition one can consider interactions where almost all the Pomeron momentum enters the hard scattering. First signs for a "super hard" Pomeron structure in $p \bar{p}$ interactions were reported by the UA8-Collaboration [3]. These effects may manifest itself in a breakdown of factorization and would lead to a delta-function like term in the Pomeron structure function. They can be explained by mechanisms called "coherent hard diffraction" [13] or by means of a direct Pomeron-quark coupling [9, 14, 15]. However the experimental information on diffractive interactions containing jets in the final state is still limited and does not allow to draw definite conclusions on the Pomeron structure.

In the present paper we discuss hard diffraction in the framework of the twocomponent Dual Parton Model [16, 17, 18, 19]. We use an ansatz developed by Capella et al. [20, 21] which is based on Regge-theory to obtain the Pomeron PDFs. Further assumptions on the normalization of the quark distributions are not necessary since this is given by $F_{2}^{\mathbb{I P}}$. Features of particle production in hard diffractive photoproduction and in hard diffractive $p \bar{p}$ interactions are investigated and compared to data [3, 5].

The paper is organized as follows. In Sect. 2 we describe the way hard diffraction is treated in our model. We especially focus on the PDFs of the Pomeron. Using Monte Carlo realizations of the model, we discuss hard diffractive particle production and calculate hard diffractive cross sections in Sect. 3. A summary is given in Sect. 4. 


\section{Description of the model}

\subsection{The triple-Pomeron approximation}

Within Regge-theory, high-mass single diffractive processes are understood by means of triple-Pomeron exchange (see Fig. 1). Using Reggeon field theory [22] this graph is treated as an usual Feynman graph. The Pomerons shown in Fig. 1 are defined by propagators $\xi_{\mathbf{I P}}(t)\left(s / s_{0}\right)^{\alpha_{\mathbf{I P}}(t)}$ and cannot be considered as particles [23]. In lowest order the differential diffractive cross section is given by the unitarity cut (Fig. 11)

$$
\frac{d^{2} \sigma_{3 \mathbb{P}}(s, t)}{d t d M^{2}}=\frac{1}{16 \pi s^{2}}\left|g_{11^{\prime}}^{\mathbb{P}}(t)\right|^{2} g_{22}^{\mathbf{P}}(0) \Gamma^{3 \mathbf{P}}(t, 0)\left|\xi_{\mathbf{P}}(t)\right|^{2}\left(\frac{s}{M^{2}}\right)^{2 \alpha_{\mathbb{P}}(t)}\left(\frac{M^{2}}{s_{0}}\right)^{\alpha_{\mathbb{P}}(0)} .
$$

With $M$ we denote the mass of the diffractively excited system. $g_{11^{\prime}}^{\mathbf{I P}}, g_{22}^{\mathbf{I P}}$, and $\Gamma^{3 \mathbf{P}}$ are the various couplings as shown in Fig. 1. $\xi_{\mathbb{P}}(t)$ is the usual signature factor

$$
\xi_{\mathbb{P}}(t)=-\frac{1+e^{-i \pi \alpha_{\mathbf{P}}(t)}}{\sin \left(\pi \alpha_{\mathbf{P}}(t)\right)} .
$$

$\alpha_{\mathbf{I P}}(t)=1+\Delta+\alpha_{\mathbf{I P}}^{\prime}(0) t$ is the Pomeron trajectory with the intercept $1+\Delta$, and $s_{0}=1 \mathrm{GeV}^{2}$. Introducing an effective Pomeron-particle cross section 23]

$$
\sigma_{\text {tot }}^{a \mathbf{P}}\left(M^{2}, t\right)=g_{22}^{\mathbf{P}}(0) \frac{\Gamma^{3 \mathbf{P}}(t, 0)}{s_{0}}\left(\frac{M^{2}}{s_{0}}\right)^{\alpha_{\mathbb{P}}(0)-1}
$$

Eq. (1) can be interpreted as a product of a Pomeron flux factor and this cross section

$$
\frac{d^{2} \sigma_{3 \mathbb{P}}(s, t)}{d t d M^{2}}=\frac{1}{16 \pi s^{2}}\left|g_{11^{\prime}}^{\mathbb{P}}(t)\right|^{2}\left|\xi_{\mathbb{P}}(t)\right|^{2} \frac{s^{2 \alpha_{\mathbb{P}}(t)}}{\left(M^{2}\right)^{2 \alpha_{\mathbb{P}}(t)-1}} \sigma_{t o t}^{a \mathbf{P}}\left(M^{2}, t\right) .
$$

To estimate the contribution of hard diffraction (an example of such a process is shown in Fig. 2) to the single diffractive cross section, $\sigma_{\text {tot }}^{a \text { IP }}$ can be replaced by the hard Pomeron-hadron/photon cross section $\sigma_{h}^{a \mathbf{P}}$. We obtain $\sigma_{h}^{a \mathbf{I P}}$ applying lowest order perturbative QCD, i.e. in case of hadron or resolved photon interactions

$\sigma_{h}^{a \mathbf{I P}}=\sum_{i, j, k, l} \frac{1}{1+\delta_{k l}} \int_{0}^{1} d x_{1} \int_{0}^{1} d x_{2} \int d \hat{t} \frac{d \sigma_{Q C D}^{i, j \rightarrow k, l}}{d \hat{t}} f_{a}^{i}\left(x_{1}, Q^{2}\right) f_{\mathbf{I P}}^{j}\left(x_{2}, Q^{2}\right) \Theta\left(p_{\perp}-p_{\perp}^{c u t o f f}\right)$

and for direct photon-Pomeron interactions

$$
\sigma_{h, d i r}^{\gamma \mathbb{P}}=\sum_{j, k, l} \int_{0}^{1} d x \int d \hat{t} \frac{d \sigma_{Q C D}^{\gamma, j \rightarrow k, l}}{d \hat{t}} f_{\mathbb{I P}}^{j}\left(x, Q^{2}\right) \Theta\left(p_{\perp}-p_{\perp}^{c u t o f f}\right) .
$$


We sum over all parton configurations and integrate having a lower cut-off in transverse momentum $p_{\perp}^{\text {cutoff }}$. Here $f_{a}^{i}\left(x, Q^{2}\right)$ are the PDFs of the hadron/photon whereas with $f_{\mathbb{I P}}^{j}\left(x, Q^{2}\right)$ we introduce distribution functions of partons inside the Pomeron. A detailed discussion of the Pomeron-PDFs will be given in the following section.

The free parameters occurring in Eq. (1), such as the proton-Pomeron coupling constant and the intercept of the Pomeron-trajectory, are obtained within the two-component Dual Parton Model by fits to data on total, elastic, and diffractive cross sections. We refer to [18, 19, 24, 25] for further details.

\subsection{Parton distributions in the Pomeron}

As shown in [9, 14, 12, 21] one can derive a Pomeron structure function $F_{2}^{\mathbb{P}}$ by relating it to the total cross section of virtual photon-proton diffractive deep inelastic scattering. Similar to the way the proton structure function $F_{2}^{p}$ can be related to $\sigma_{\text {tot }}^{\gamma^{*} p}$ one gets

$$
F_{2}^{\mathbf{P}}\left(x, Q^{2}, t\right)=\frac{Q^{2}}{4 \pi^{2} \alpha_{e m}} \sigma_{t o t}^{\gamma^{*}} \mathbf{P}\left(M^{2}, Q^{2}, t\right), \quad x=\frac{Q^{2}}{Q^{2}+M^{2}}
$$

Convoluting $F_{2}^{\mathbb{P}}$ with a Pomeron-flux factor, one obtains the structure function of diffractive dissociation. Using Regge factorization it is possible to calculate $F_{2}^{\mathbb{I P}}$ from the deuteron structure function $F_{2}^{d}$. The complete formalism is given in Ref. [20, 21]. Here we only want to give the main formulas which are necessary to understand the PDFs of the Pomeron we will use afterwards. The proton structure function is parametrized at moderate values of $Q^{2}\left(Q^{2} \leq 5 \mathrm{GeV}^{2}\right)$ 20]

$$
\begin{aligned}
& F_{2}^{p}\left(x, Q^{2}\right)=S\left(x, Q^{2}\right)+V\left(x, Q^{2}\right)= \\
& A x^{-\Delta\left(Q^{2}\right)}(1-x)^{n\left(Q^{2}\right)+4}\left(\frac{Q^{2}}{Q^{2}+a}\right)^{1+\Delta\left(Q^{2}\right)} \\
& +B x^{1-\alpha_{R}}(1-x)^{n\left(Q^{2}\right)}\left(\frac{Q^{2}}{Q^{2}+b}\right)^{\alpha_{R}}
\end{aligned}
$$

with

$$
\Delta\left(Q^{2}\right)=\Delta_{0}\left(1+\frac{2 Q^{2}}{Q^{2}+d}\right), \quad n\left(Q^{2}\right)=\frac{3}{2}\left(1+\frac{Q^{2}}{Q^{2}+c}\right) .
$$

We again refer to [20, 21] for the exact values of the parameters entering the expressions and a detailed discussion of the $Q^{2}$-dependent intercept. The Pomeron structure function can be related to the deuteron structure function by the following substitutions

$$
F_{2}^{\mathbf{I P}}\left(x, Q^{2}, t\right)=F_{2}^{d}\left(x, Q^{2} ; A \rightarrow e(t) A, B \rightarrow f(t) B, n\left(Q^{2}\right) \rightarrow n\left(Q^{2}\right)-2\right) .
$$


$e$ and $f$ are ratios of coupling constants. The $t$-dependence of the Pomeron$\mathrm{PDF}$ is completely given by the ratios $e$ and $f$. We use $e(0)=3 f(0)=0.1$ [21] and an exponential dependence on $t$ with the slope $b=0.5 \mathrm{GeV}^{-2}$ [23, 19, 26]. The substitution $n\left(Q^{2}\right) \rightarrow n\left(Q^{2}\right)-2$, for example, is due to the similarity of the valence quark distribution in the Pomeron and a meson. The first term in Eq. (8) determines the sea-quark distribution whereas the last term is responsible for the behaviour of the valence quark distribution. Using Eq. (10), and the definition

$$
F_{2}^{\mathbf{I P}}\left(x, Q^{2}, t\right)=\sum_{q} e_{q}^{2} x\left(f_{\mathbf{I P}}^{q}\left(x, Q^{2} ; t\right)+f_{\mathbf{I P}}^{\bar{q}}\left(x, Q^{2} ; t\right)\right)
$$

where $e_{q}$ are the corresponding quark-charges, we obtain at moderate $Q^{2}$-values for the Pomeron PDFs

$$
\begin{aligned}
x f_{\mathbf{P}}^{u}\left(x, Q^{2} ; t\right) & =x f_{\mathbb{P}}^{\bar{u}}\left(x, Q^{2} ; t\right)=x f_{\mathbf{P}}^{d}\left(x, Q^{2} ; t\right)=x f_{\mathbf{P}}^{\bar{d}}\left(x, Q^{2} ; t\right) \\
& =\frac{3}{4} e(t) S\left(x, Q^{2}\right)+\frac{9}{10} f(t) V\left(x, Q^{2}\right) \\
x f_{\mathbf{P}}^{s}\left(x, Q^{2} ; t\right) & =x f_{\mathbb{P}}^{\bar{s}}\left(x, Q^{2} ; t\right)=\frac{3}{4} e(t) S\left(x, Q^{2}\right) .
\end{aligned}
$$

For simplicity, we assume a $\mathrm{SU}(3)$-symmetrical sea of light quarks. The charmquark distribution of the Pomeron is suppressed at the $Q^{2}$-values considered so far. In contrast to the nucleon it is not possible to determine the normalization of the gluon distribution in the Pomeron by momentum sum rule. We are therefore free to choose a specific form and normalization. With respect to the experimental observations which favor a relatively "hard" structure we use

$$
x f_{\mathbb{P}}^{g}\left(x, Q_{0}^{2} ; t\right)=K\left(Q_{0}^{2}, t\right) x(1-x)
$$

where $K\left(Q_{0}^{2}, t\right)$ depends on the normalization chosen. In the following we take the normalization of the gluon distribution according to the scaling factor resulting for the quark distribution from Eq. (10).

As already mentioned all distributions obtained are limited to $Q^{2} \leq 5 \mathrm{GeV}^{2}$. In order to get PDFs of the Pomeron at higher $Q^{2}$-values we take (12) and (13) as input distributions for a QCD evolution in the leading logarithmic approximation [12]. The evolution was done using the code of [27, 28] with $Q_{0}^{2}=2 \mathrm{GeV}^{2}$. The result is shown in Fig. 3 and 4 , where we have plotted $x f_{\mathbf{P P}}^{u}, x f_{\mathbf{I P}}^{s}$, and $x f_{\mathbf{I P}}^{g}$ for different values of $Q^{2}$. Within this formalism, the normalization of the Pomeron PDFs is given by

$$
N_{q}=\sum_{q, \bar{q}} \int_{0}^{1} d x x f_{\mathbf{P}}^{q}\left(x, Q^{2}, t\right), \quad N_{g}=\int_{0}^{1} d x x f_{\mathbf{P}}^{g}\left(x, Q^{2}, t\right)
$$

which is shown for $t=0 \mathrm{GeV}^{2}$ in Fig. 5. The hardness of the $u$ - and $d-$ quark distributions at low values of $Q^{2}$ is governed by the valence part of (10), i.e. 
for $x>0.5$ we are mainly dealing with a $\sqrt{x}(1-x)^{n\left(Q^{2}\right)-2}$-behaviour, $n\left(Q^{2}\right)$ being about 2.0...3.0 ( $c=3.55$ [20]). We note that this behaviour is similar to the $Q^{2}$-independent predictions of [9, 14], whereas at high $Q^{2}$-values our quark-distributions become softened due to the sea-quark contribution and the QCD-evolution.

Furthermore, we show in Fig. 6 the structure function $F_{2}^{\mathbb{I P}}$ for different $Q^{2}$ scales. Again, the flat shape of $F_{2}^{\mathbb{P P}}$ is determined by the valence quark distributions.

\section{Particle production in hard diffractive inter- actions}

\subsection{Sampling of hard single diffractive events}

The Monte-Carlo treatment of soft single diffractive hadron-hadron interactions within the two-component DPM is described in [17, 18]. The generation of single diffractive events in photoproduction will be discussed elsewhere [29]. Here we want to focus on the way the existing models have been extended to diffractive jet-production. This has been done for $p \bar{p}$ collisions using an extension to the DTUJET-93 code [30] and for $p \bar{p}$ as well as $\gamma p$ collisions using the DTUJETPHOJET code [31, 29].

The Monte-Carlo implementation is similar to that of usual hard scattering processes (i.e. processes involving high $p_{t}$ ) between hadrons or between hadrons and photons. The main differences are: (i) the interaction is boosted to the rest system of the diffractively excited "blob", the CM energy is therefore given by the diffractive mass, and (ii) one hadron is replaced by a Pomeron with a virtuality $t$. The momenta of the partons entering the hard $2 \longrightarrow 2$ scattering process are obtained using conventional PDFs of hadrons and photons [32, 33, 34] and the PDFs of the Pomeron introduced in the previous section. Initial state radiation which significantly modifies the multiparticle final state of interactions involving high transverse momenta has been implemented. The chain system to be hadronized using JETSET 7.3 [35, 36] is determined by the color flow taking cross sections for different color flow diagrams into consideration [37, 38].

Parts of the MC-realization of hard diffraction are technically similar to the ones described in [39, 40], which were so far mainly used to understand the underlying interactions. However, we would like to emphasize that our starting points are completely different. Our investigation is based on the two-component DPM which treats soft and hard scattering processes in an unified manner. The free parameters are fixed by fits to cross section data. Similar to [39, 40] we assume hard diffraction to be based on a partonic structure of the Pomeron but we start from a Pomeron structure function which is completely determined by fits to data on the proton structure function and a ratio of coupling constants which 
follows from the model. There is no further freedom in choosing a specific $x$ - and $Q^{2}$-dependence of the quark distribution inside the Pomeron. The normalizations of the Pomeron PDFs (and therefore the hard diffractive cross sections) are obtained from the model rather than imposing additional assumptions [39, 40].

\subsection{Hard diffraction in photoproduction}

Recent measurements at the electron proton collider HERA at DESY have shown that a substantial part of minimum bias photoproduction [5, (7] and deep inelastic scattering [ [4, [] events exhibits diffractive features similar to hadron-hadron scattering. First distributions of so called rapidity gap events have been published [5. 7], but the data are not yet corrected for acceptance and do not allow absolute comparisons. We will apply the model developed to investigate diffraction dissociation of photons in quasi-real photon-hadron scattering and we understand that more data will be published soon.

The simulation of diffractive events in photoproduction can be done similar to diffraction in hadron-hadron scattering substituting the hadron-Pomeron scattering subprocess by photon-Pomeron scattering. In the calculation of the total diffractive cross section, the absorptive corrections due to multiple photon-hadron scattering are taken into account [19]. For the simulation of photon-Pomeron scattering we neglect unitarity corrections (e.g. multiple photon-Pomeron scattering) which become important only for very high diffractive masses?. Thus, the simulation of hard photon-Pomeron scattering follows directly from Eq. (5.,6). The Pomeron flux is calculated using Eq. (11).

To calculate $e p$ photoproduction cross sections, the flux of quasi-real photons has to be estimated. Here we apply the improved Weizsäcker-Williams approximation 41

$$
f_{e / \gamma}(y)=\frac{\alpha}{2 \pi}\left(\frac{1+(1+y)^{2}}{y} \ln \frac{Q_{\max }^{2}}{Q_{\min }^{2}}-\frac{1(1-y)}{y}\right)
$$

with the kinematical cuts

$$
0.25<y<0.7 \quad Q_{\min }^{2}=3 \cdot 10^{-8} \mathrm{GeV}^{2} \quad Q_{\max }^{2}=10^{-2} \mathrm{GeV}^{2} .
$$

$y$ and $Q^{2}$ denote the energy fraction taken by the photon from the electron and the photon virtuality, respectively.

Using our Monte Carlo program, complete hadronic final states have been generated and analyzed. According to the experimental conditions only events passing the $\eta_{\text {max }}$-cut [42, 6] have been accepted for the further analysis. In addition, a few less restrictive cuts have been applied to match the experimental selection procedure described in [5].

In Fig. 0 we show the transverse momentum distribution of charged particles for the selected rapidity gap events in the pseudorapidity range $-1.5<\eta_{l a b}<1.5$.

\footnotetext{
${ }^{*}$ High diffractive masses are suppressed by applying the experimental cuts.
} 
The absolute cross sections obtained with the model are given. Together with the predictions we show uncorrected data of the H1 Collaboration [5] on charged tracks. It is expected that the shape of this distribution can be compared with calculations for transverse momenta higher than $1 \mathrm{GeV} / \mathrm{c}$ 43]. Note that the data are scaled in order to compare their shape to our calculation. The systematic difference between the model and the calculation at low $p_{\perp}$ can be qualitatively explained by the $p_{\perp}$-dependent experimental acceptance in this region [43]. In addition, the contribution from direct photon processes is shown.

Using a cone jet algorithm similar to the one used in [5] the transverse energy and pseudorapidity distributions of jets with $E_{t}>4 \mathrm{GeV}$ are calculated. In Fig. 8 we show the transverse energy distribution of jets together with uncorrected $\mathrm{H} 1$ data [5]. Since the so called jet-pedestal is small in diffractive events [5, 0, 44] the transverse jet distribution should not be drastically influenced by acceptance effects. In Fig. 9 the corresponding pseudorapidity distribution of jets is compared to $\mathrm{H} 1$ data. In both figures, the H1 data are again scaled to compare their shape with the model predictions.

\subsection{Hard diffractive proton-antiproton interactions}

\subsubsection{Cross sections for hard single diffraction}

An estimation of cross sections for hard diffractive events containing jets in the final state can be obtained using Regge-factorization (Eq. (田) together with the hard Pomeron-particle cross sections given in Eq. (5) and (6). However the cross sections depend strongly on the partonic cut-off in transverse momentum which enters the integrations. In addition, further uncertainties arise from choosing a certain scale in (5) and (6) and from the definition of a diffractive event itself, i.e. from the $t$ - and $M^{2}$-ranges the differential hard diffractive cross section has to be integrated over.

Reliable predictions for cross sections in hard diffraction can therefore only be given for a certain experimental set-up taking into account all kinematical cuts applied and jet rates based on jet-finding algorithms which were used to obtain the experimental results. This has been done for the experimental set-up of UA8 [3] with a jet-finding algorithm which will be described further below. We calculate the ratio of the hard diffractive cross section and the total diffractive cross section using the MRS $\mathrm{D}^{\prime}$ [32] set for the parton distributions in the proton/antiproton and $g_{p \mathbb{P}}(0)=6.2 \sqrt{m b}, \Gamma^{3 \mathbb{P}}(t=0)=0.08 \sqrt{m b} \mathrm{GeV}^{2}$, $\Delta=0.078, \alpha^{\prime}(0)=0.25 \mathrm{GeV}^{-2}$ and $Q^{2}=p_{\perp}^{2}$ [19]. To obtain ratios which correspond to the UA8-cuts we multiply these values by the fraction of those events which contain at least two jets of a transverse energy $E_{t}^{\text {jet }} \geq E_{t}^{\text {cutoff }}$ and of a pseudorapidity $|\eta|<2$ in their final state. Experimental data for these ratios in preliminary form were given in [45], final values will be available soon [46] and we understand, that our calculations shown in Fig. 10 will be consistent with these 
data [47.

\subsubsection{Hard diffractive particle production}

Hard diffractive proton-antiproton interactions were recently investigated by UA8-Collaboration [3] at a CM energy of $630 \mathrm{GeV}$. Only limited information on absolute hard diffractive cross sections is available so far 45 (see previous section). It is therefore not possible to compare our model directly to these data. However, since our model is able to describe data on hard diffraction in

photoproduction rather well it might be worthwhile to show also predictions for hard diffractive $p \bar{p}$-interactions. In particular we apply the same cuts to the final state as they were used in the experiments [3]. Jets are identified using a cone-algorithm in the $\eta-\phi$-plane, with $\phi$ being the azimuthal angle and $|\eta|<2$. If a jet with $E_{t}>8 \mathrm{GeV}$ is found the search for its axis is iterated calculating $E_{t}-$ weighted sums over cells within an unit-cone radius. According to the UA8data all distributions discussed in this section are normalized to unit area. Our results are obtained with the PDF set MRS D0' for the parton distributions in the proton/antiproton.

As mentioned in [3] a variable sensitive to the partonic structure of the Pomeron could be $x_{\text {jet }}$. It is defined as the longitudinal momentum component of a jet normalized to its maximum value in the Pomeron-antiproton CM system. In Fig. 11 we present predictions of our model for momentum fractions of the quasi-elastically scattered proton $x_{p}$ between 0.92 and 0.94 . As stated by UA8 [3] the data were obtained with essentially full acceptance at positive $x_{j e t}$-values, i.e. it can be expected that our calculations agree well with the data also after the corrections have been applied.

In Fig. 12 we show the distribution of the jet-pseudorapidity in the antiprotonproton CM system again for the same $x_{p}$-bin. The tail at positive $\eta$-values is influenced mainly by the structure of the Pomeron.

A variable which may indicate whether there is a "super hard" Pomeron structure is $x_{2 j e t s}$ - the longitudinal momentum of a two-jet system, again normalized to its maximum value in the Pomeron-antiproton system. The results of our calculation are plotted in Fig. 13 showing the contribution from quarks and gluons of the Pomeron-PDF separately. Due to the shape of the valence quark distribution (see Sect. 2.2) the quarks mainly contribute to higher values of $x_{2 j e t s}$ whereas the gluon contribution is peaked around 0.2. Using our Pomeron-PDF we therefore obtain a significant fraction of events containing 2-jet systems with $x_{2 j e t s}>0.7$. Considering the reasonable agreement found with the $\gamma p$ data the question to which extent a "super hard" contribution is still necessary within our model cannot be answered before corrected measurements are available. 


\section{Summary and conclusions}

Hard diffraction in hadron-hadron collisions and in photoproduction has been investigated in the framework of the two-component DPM. Since soft diffractive interactions at collider energies are well-described in terms of Pomeron exchange processes, diffractive jet-production may provide new information on the Pomeron-structure [1].

In the present paper we study features of hard diffractive particle production treating the Pomeron as a partonic object. PDFs of the Pomeron are obtained from a Pomeron structure function motivated by Regge-theory. Whereas the quark distributions follow directly from the parametrization of the Pomeron structure function, it is possible to choose an ansatz for the gluon distribution which accounts for the experimentally observed hard Pomeron structure. The normalizations of the quark distributions of the Pomeron are determined by the scaling factor relating the Pomeron structure function to the deuteron structure function. The Pomeron PDFs are evolved to high values of $Q^{2}$ applying leading logarithmic QCD evolution equations. Using the triple-Pomeron approximation, we are able to give predictions on absolute cross sections and distributions.

We demonstrate that our model and the Pomeron PDFs used are in reasonable agreement with presently available data on hard diffraction in $\gamma p$ collisions from the HERA-collider. Since there are no absolute distributions published the comparison was restricted to the shape.

Cross sections for hard diffraction depend strongly on kinematical cuts and on assumptions defining an event as being produced diffractively. Predictions can therefore only be given taking a specific experimental set-up into account.

A comparison of hard diffractive particle production in hadron-hadron interactions to data is presently limited to data published by the UA8-Collaboration which do not allow absolute comparisons. Nevertheless, the results obtained within our model look promising and may explain the main features of these processes. We are not yet able to draw any conclusions concerning a possible "super hard" Pomeron structure. Further investigations will be necessary as soon as more data become available.

\section{Acknowledgements}

The authors acknowledge stimulating discussions with F.W. Bopp, A. Capella, C. Merino, D. Pertermann, and P. Schlein. We would like to thank P. Aurenche providing us the QCD-evolution code. We are grateful to G. Ingelman and P. Schlein for helpful discussions concerning the UA8 data. One of the authors (R.E.) is indebted to A. Rostovstev (H1 Collaboration) for many discussions and valuable information on the HERA data.

\footnotetext{
${ }^{\dagger}$ The code is available on request from the authors (email: sroesler@cernvm.cern.ch).
} 


\section{References}

[1] G. Ingelman and P. E. Schlein: Phys. Lett. B152 (1985) 256

[2] UA8 Collab.: R. Bonino et al.: Phys. Lett. B211 (1988) 239

[3] UA8 Collab.: A. Brandt et al.: Phys. Lett. B297 (1992) 417

[4] H1 Collab.: T. Ahmed et al.: Nucl. Phys. B429 (1994) 477

[5] H1 Collab.: T. Ahmed et al.: Observation of hard processes in rapidity gap events in $\gamma p$ interactions at HERA, DESY 94-198, to be published in Nucl. Phys. , 1994

[6] ZEUS Collab.: M. Derrick et al.: Phys. Lett. B315 (1993) 481

[7] ZEUS Collab.: M. Derrick et al.: Observation of hard scattering in photoproduction events with a large rapidity gap at HERA, DESY 94-210, 1994

[8] E. L. Berger, J. C. Collins, D. E. Soper and G. Sterman: Nucl. Phys. B286 (1987) 704

[9] A. Donnachie and P. V. Landshoff: Phys. Lett. B191 (1987) 309

[10] A. Donnachie and P. V. Landshoff: Nucl. Phys. B303 (1988) 634

[11] G. Ingelman and K. Prytz: Z. Phys. C58 (1993) 285

[12] N. N. Nikolaev and B. G. Zakharov: Z. Phys. C53 (1992) 331

[13] J. Collins, L. Frankfurt and M. Strikman: Phys. Lett. B307 (1993) 161

[14] A. Donnachie and P. V. Landshoff: Nucl. Phys. B244 (1984) 322

[15] B. Kniehl, H.-G. Kohrs and G. Kramers: Diffractive photoproduction of jets with a direct Pomeron coupling at HERA, DESY 94-140, 1994

[16] A. Capella, U. Sukhatme, C. I. Tan and J. Tran Thanh Van: Phys. Rep. 236 (1994) 227

[17] P. Aurenche, F. W. Bopp, A. Capella, J. Kwiecinski, M. Maire, J. Ranft and J. Tran Thanh Van: Phys. Rev. D45 (1992) 92

[18] S. Roesler, R. Engel and J. Ranft: Z. Phys. C59 (1993) 481

[19] R. Engel: Photoproduction within the two-component Dual Parton Model: Amplitudes and Cross Sections, Siegen University SI 94-02, to be published in Z. Phys. C, 1994 
[20] A. Capella, A. Kaidalov, C. Merino and J. Tran Thanh Van: Phys. Lett. B337 (1994) 358

[21] A. Capella, A. Kaidalov, C. Merino and J. Tran Thanh Van: Diffractive dissociation in deep inelastic scattering at HERA, LPTHE Orsay 94-42, to be published in Phys. Lett. B, 1994

[22] M. Baker and K. A. Ter-Martirosyan: Phys. Rep. 28C (1976) 1

[23] A. B. Kaidalov: Phys. Rep. 50 (1979) 157

[24] F. W. Bopp, R. Engel, D. Pertermann and Ranft: Phys. Rev. D49 (1994) 3236

[25] R. Engel, F. W. Bopp, D. Pertermann and J. Ranft: Phys. Rev. D46 (1992) 5192

[26] A. Capella, J. Tran Thanh Van and J. Kaplan: Nucl. Phys. B97 (1975) 493

[27] A. Devoto, D. W. Duke and J. F. Owens: Phys. Rev. D27 (1983) 508

[28] P. Aurenche, R. Baier, M. Fontannaz, M. N. Kienzle-Focacci and M. Werlen: Phys. Lett. B233 (1989) 517

[29] R. Engel: in preparation,

[30] F. W. Bopp, R. Engel, D. Pertermann, J. Ranft and S. Roesler: Comput. Phys. Commun. 83 (1994) 107

[31] R. Engel: Photoproduction within the Dual Parton Model, to be published in Proceedings of Rencontre de Moriond, Meribel, 1994

[32] A. D. Martin, R. G. Roberts and W. J. Stirling: Phys. Lett. B306 (1993) 145

[33] M. Glück, E. Reya and A. Vogt: Phys. Rev. D46 (1992) 1973

[34] H. Plothow-Besch: Comput. Phys. Commun. 75 (1993) 396

[35] T. Sjöstrand: Comput. Phys. Commun. 39 (1986) 347

[36] T. Sjöstrand and M. Bengtsson: Comput. Phys. Commun. 43 (1987) 367

[37] B. L. Combridge, J. Kripfganz and J. Ranft: Phys. Lett. B70 (1977) 234

[38] H. U. Bengtsson: Comput. Phys. Commun. 31 (1984) 323 
[39] P. Bruni and G. Ingelman: Diffractive hard scattering at ep and $p p$ colliders, Talk given at Marseille HEP Conference, in Proccedings of International European Conference on High Energy Physics, Page 595, ed. by J. CarrM. Perrottet, Edition Frontieres, Gif-sur-Yvette, 1994

[40] H. Jung: Hard diffractive scattering in high energy ep collisions and the Monte Carlo generator RAPGAP, DESY 93-182, submitted to Comput. Phys. Commun. , 1993

[41] S. Frixione, M. Mangano, P. Nanson and G. Ridolfi: Phys. Lett. B319 (1993) 339

[42] H1 Collab.: T. Ahmed et al.: Phys. Lett. B299 (1993) 374

[43] A. Rostovtsev: private communication,

[44] P. E. Schlein: Talk given at the 14th Meeting of the Large Hadron Collider Committee, CERN, 1994

[45] P. E. Schlein: The evidence for partonic behaviour of the Pomeron, preprint UCLA-PPh0061, Talk given at Marseille HEP Conference, in Proccedings of International European Conference on High Energy Physics, Page 592, ed. by J. Carr-M. Perrottet, Edition Frontieres, Gif-sur-Yvette, 1994

[46] UA8 Collab.: A. Brandt et al.: Cross section measurement of hard diffraction at the SPS-Collider, in preparation, 1995

[47] P. E. Schlein: private communication, 


\section{Figure Captions}

1. A cut triple-Pomeron graph (a) describes multiparticle final states characterized by a rapidity gap (b).

2. Example for a hard diffractive scattering process. A gluon of the Pomeron undergoes a hard scattering with a gluon of the lower particle labeled with 2 .

3. $u^{-}$and $s^{-}$-quark distributions in the Pomeron are plotted for different values of $Q^{2}$. The initial distribution of the QCD-evolution is shown for $Q_{0}^{2}=$ $2 \mathrm{GeV}^{2}$.

4. Gluon distribution in the Pomeron shown for different values of $Q^{2}$.

5. Normalization of the quark and gluon distribution function of the Pomeron.

6. Pomeron structure function $F_{2}^{\mathbb{P}}\left(x, Q^{2} ; t=0\right)$.

7. Inclusive charged particle cross section for particle with $\left|\eta_{l a b}\right|<1.5$. The model predictions are shown as full line and compared to H1 data (see text).

8. Inclusive transverse energy distribution of jets calculated for jets with $\left|\eta_{\text {jet }}\right|<$ 1.5 and compared to H1 data.

9. Inclusive pseudorapidity distribution of jets in rapidity gap events with $E_{t, j e t}>4 \mathrm{GeV}$ calculated with the model and shown with $\mathrm{H} 1$ data.

10. Ratio of hard diffractive cross sections for two-jet events and total diffractive cross sections. According to forthcoming the UA8 data 47, 46, to whom our calculation might be compared, a lower cut in transverse energy of $8 \mathrm{GeV}$ has been applied.

11. Distribution of the longitudinal momenta of jets normalized to their maximum value in the CM system of the Pomeron-proton interaction. The predictions of the model (labeled "MC") are shown for a momentum fraction of the quasi-elastically scattered proton between 0.92 and 0.94 . The uncorrected UA8-data [3] are plotted separately (labeled "UA8").

12. Pseudorapidity distributions of jets in the CM system of the proton-antiproton interaction. The Monte Carlo results (MC) are shown for a momentum fraction of the quasi-elastically scattered proton between 0.92 and 0.94 . The uncorrected UA8-data [3] are plotted separately.

13. Distributions of the total longitudinal momentum of a two-jet system normalized to the maximum value for the quark and gluon contribution of the Pomeron and both contributions together are shown. Separately we plot the uncorrected UA8-data [3]. 


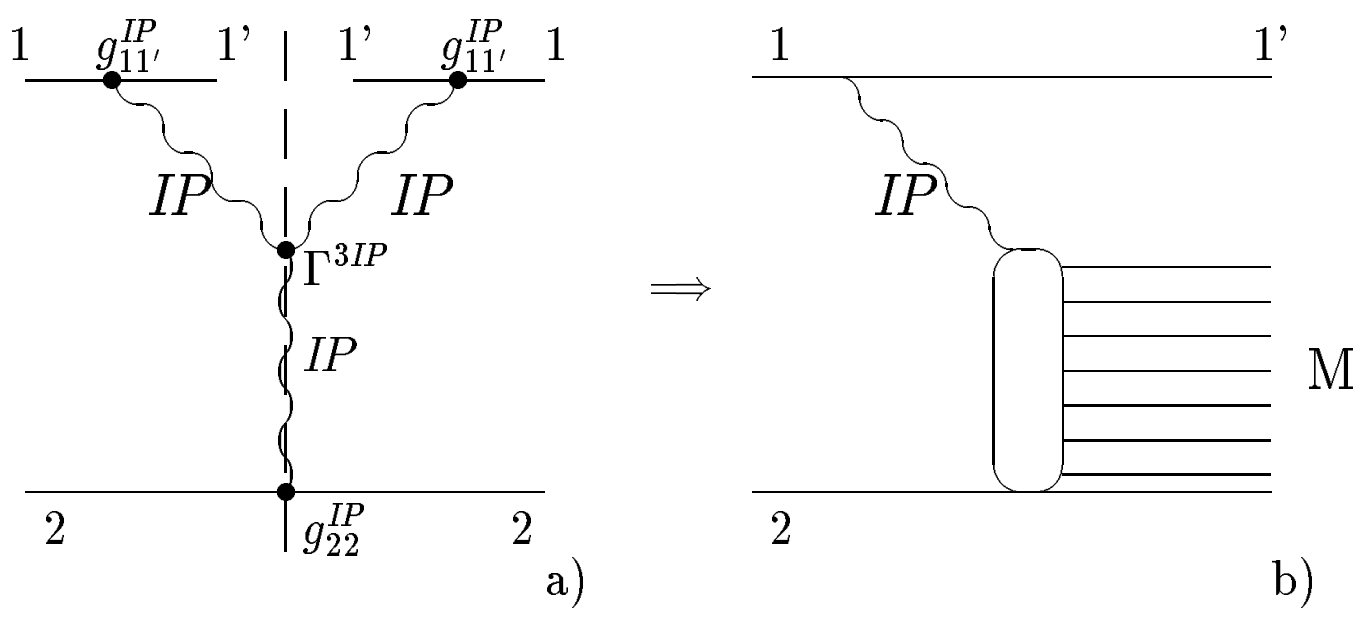

Fig. 1

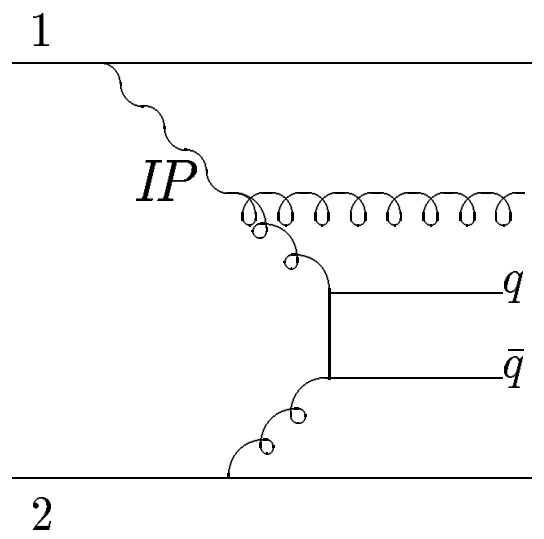

Fig. 2 

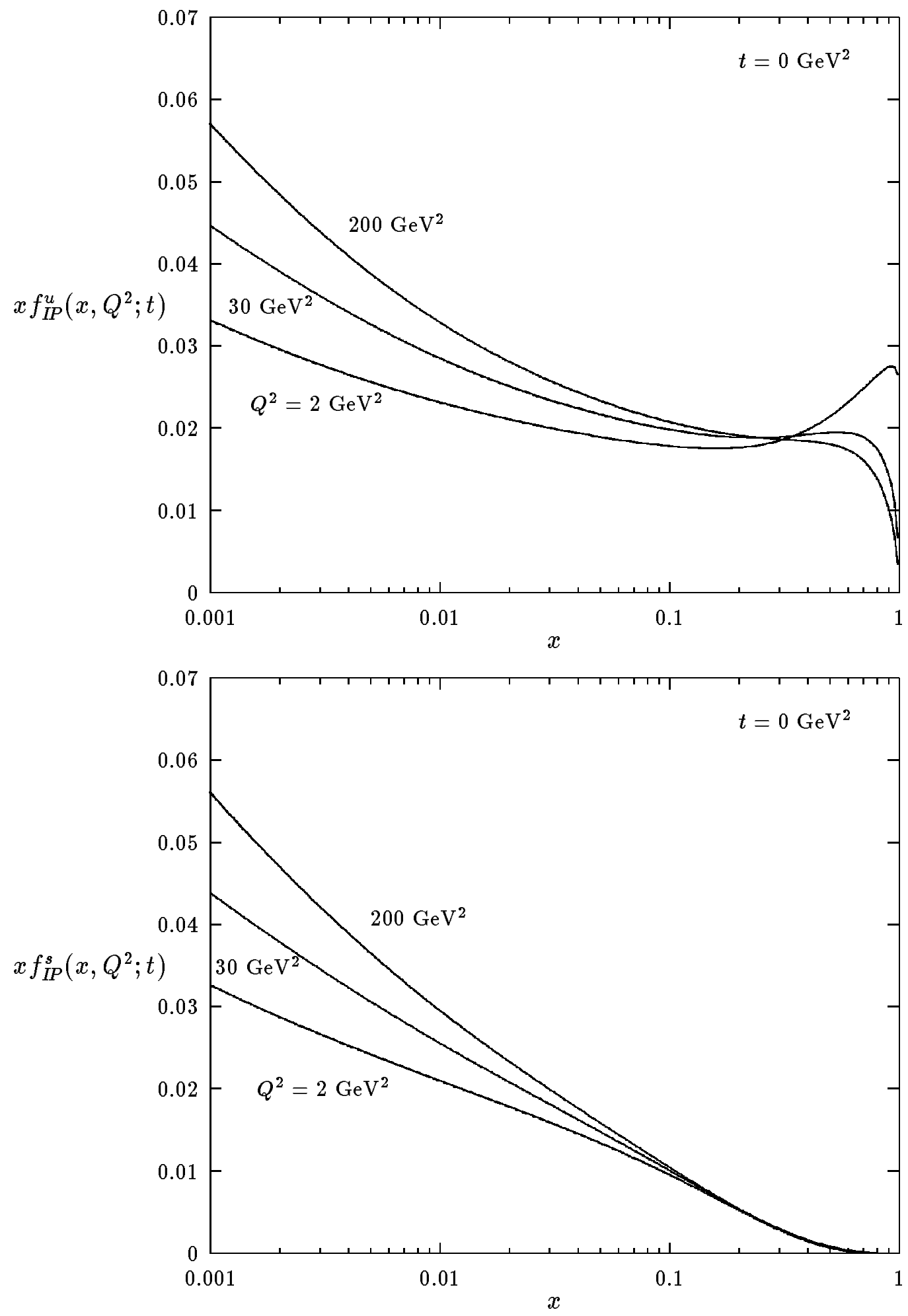

Fig. 3 


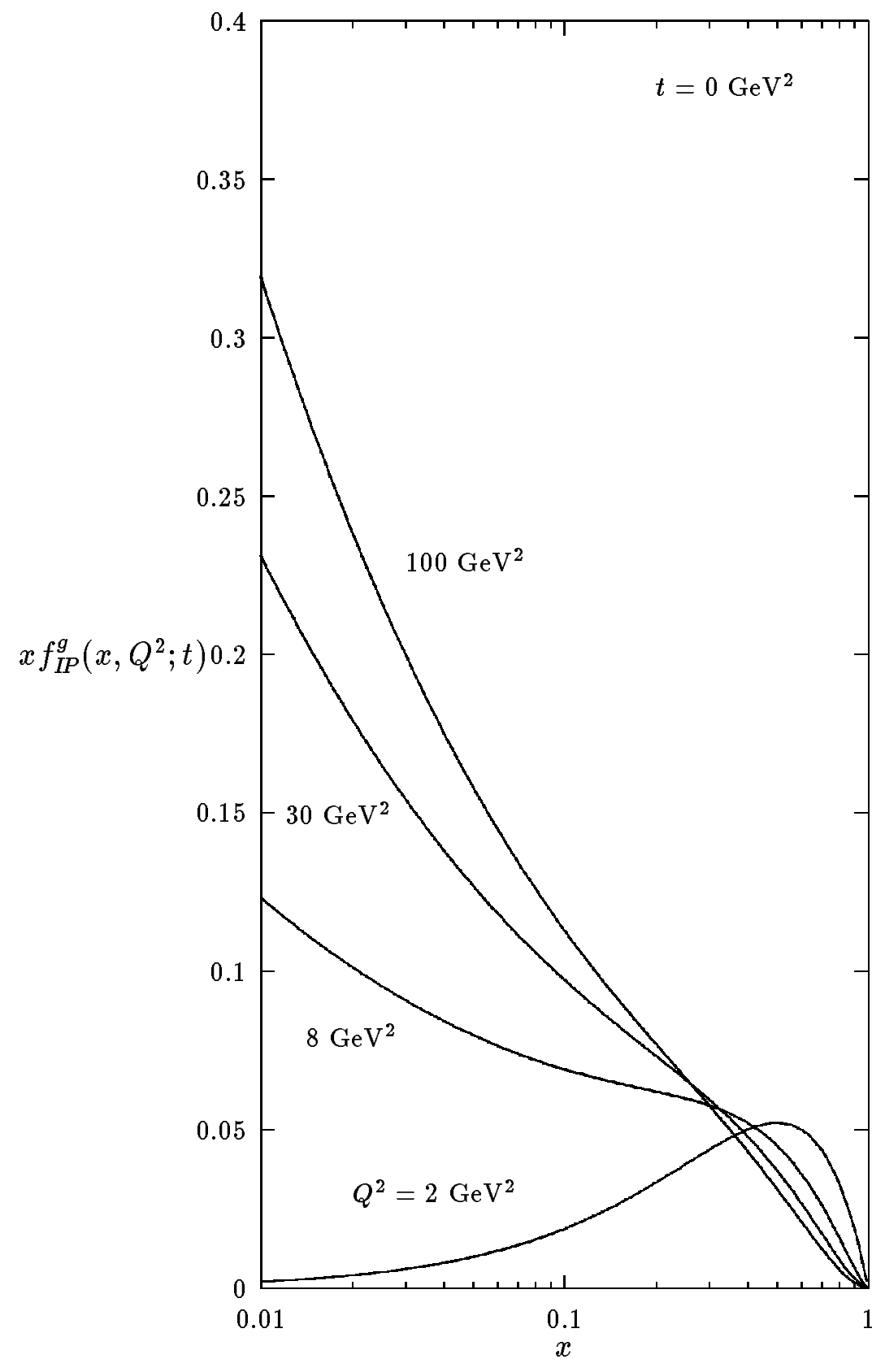

Fig. 4 


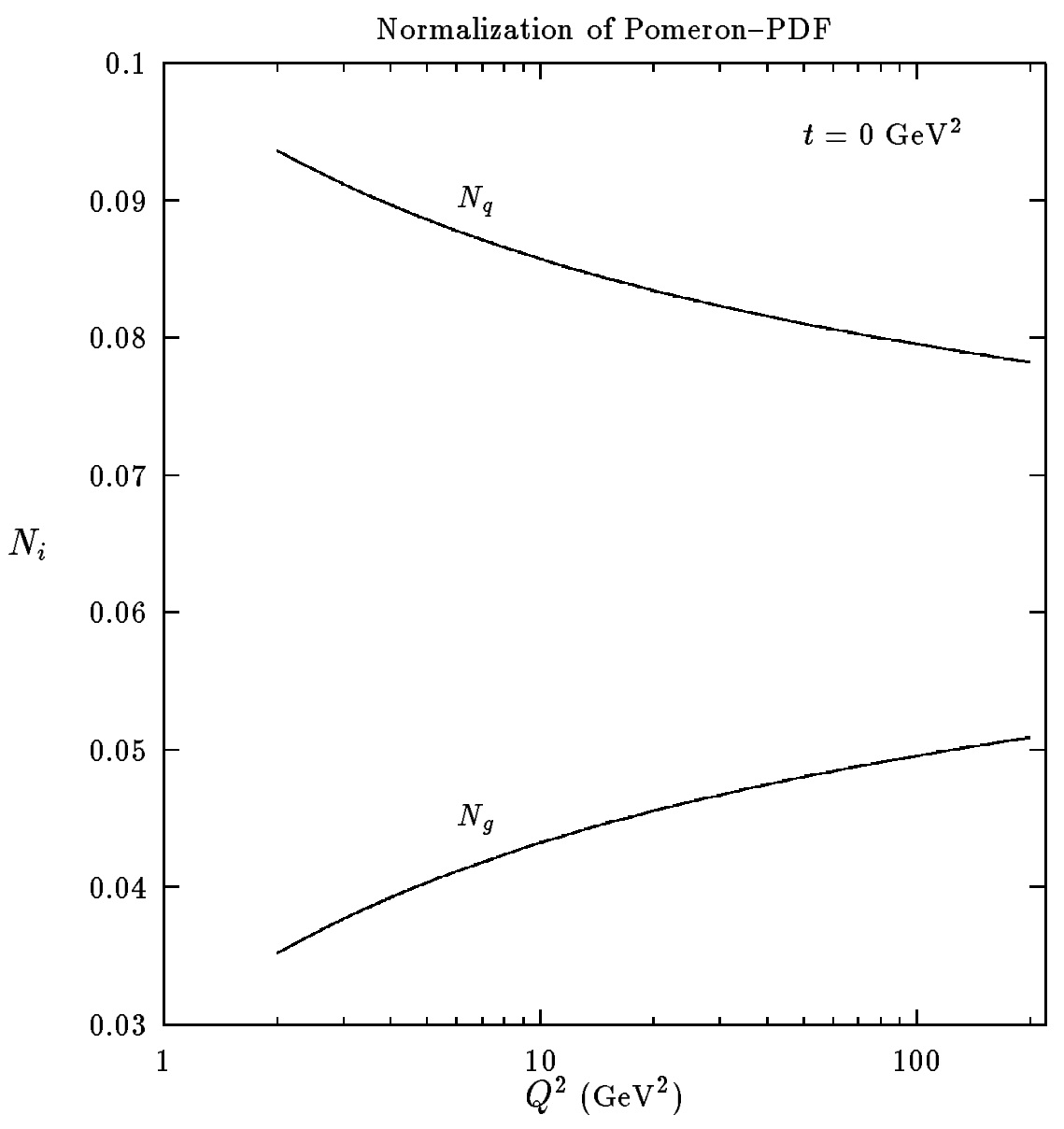

Fig. 5 


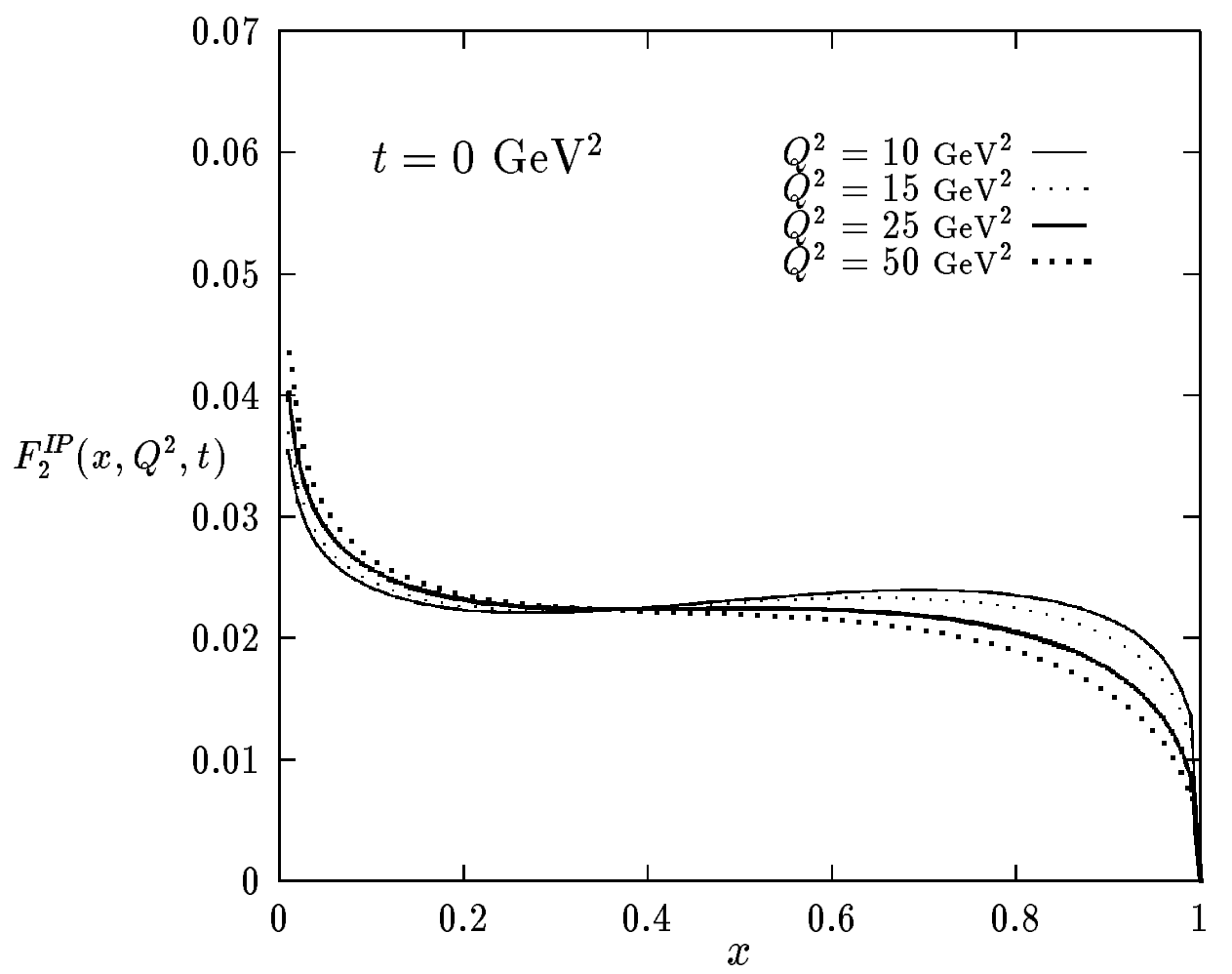

Fig. 6 


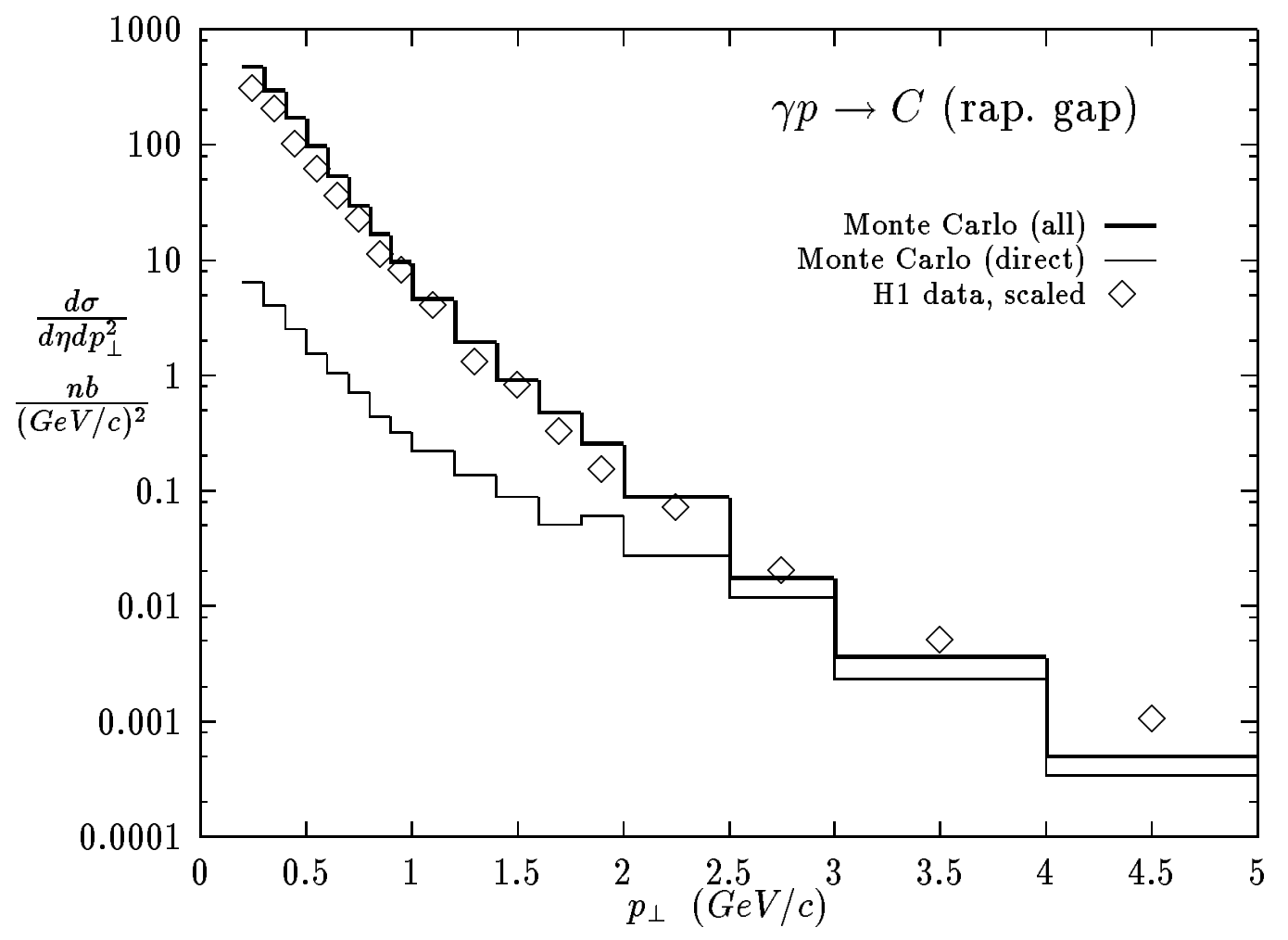

Fig. 7 


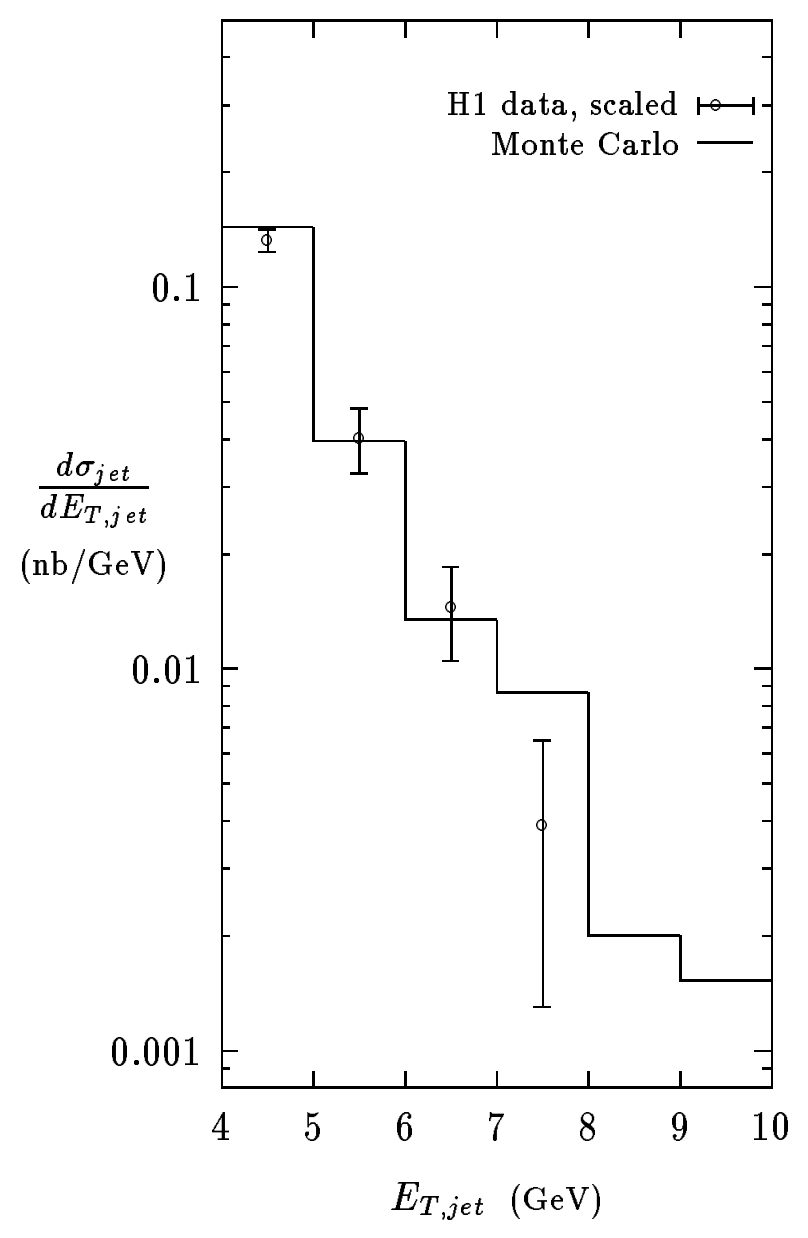

Fig. 8

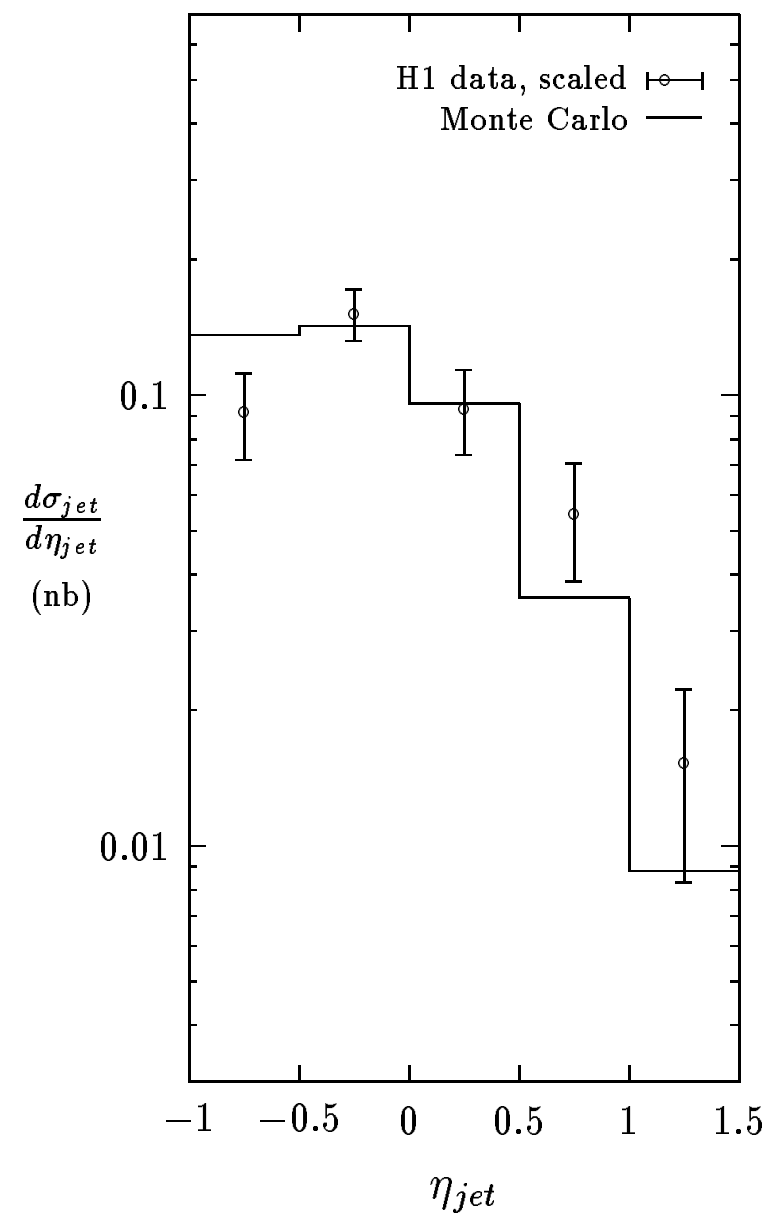

Fig. 9 


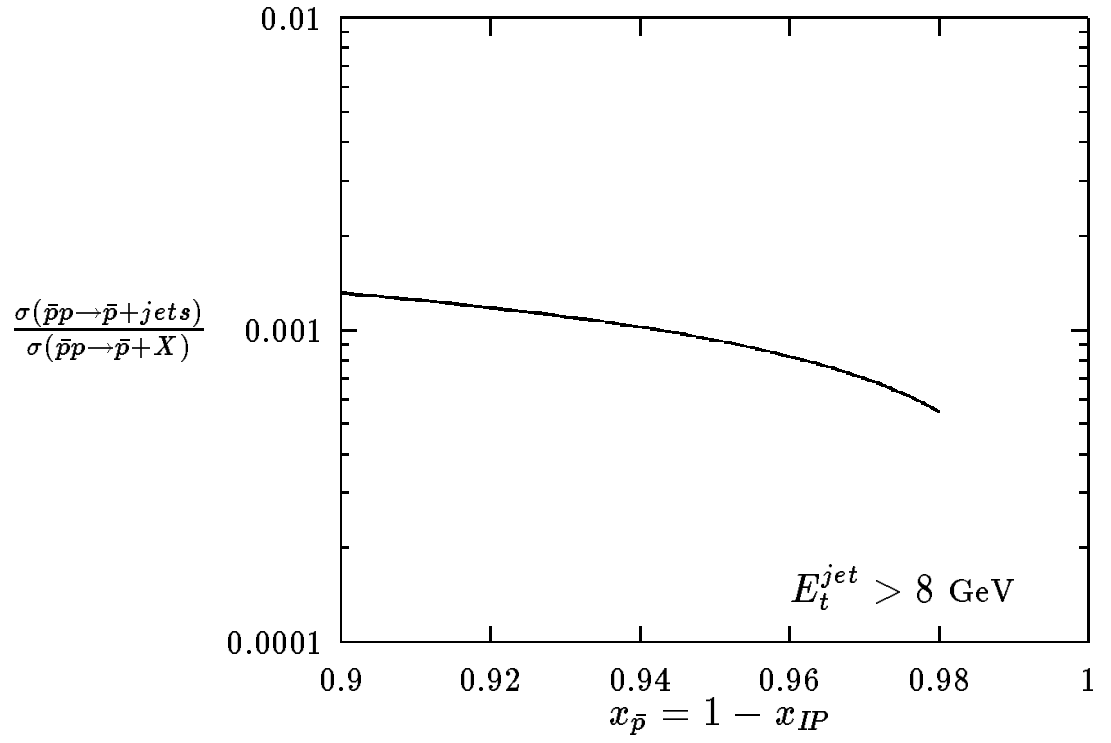

Fig. 10 

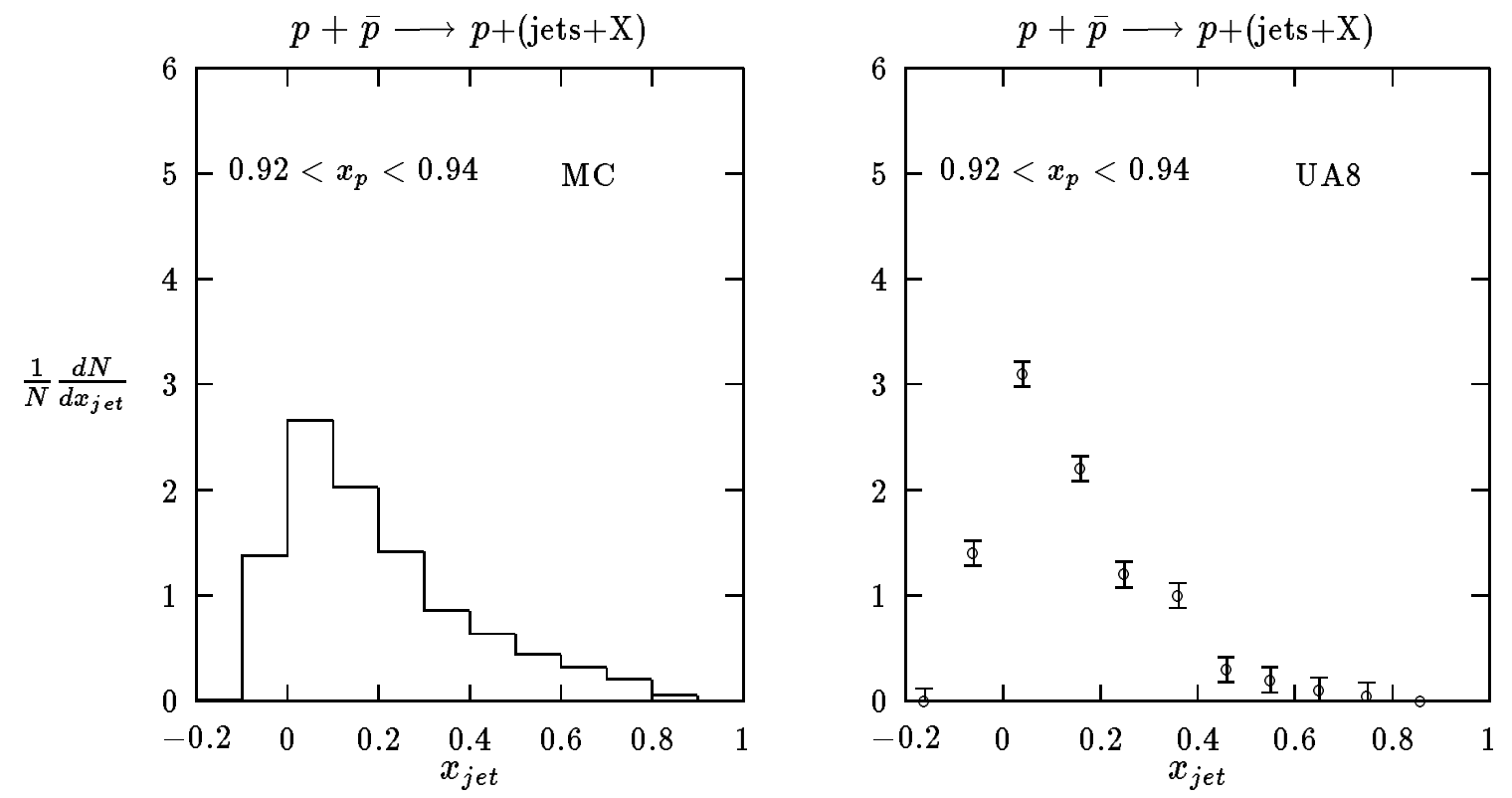

Fig. 11
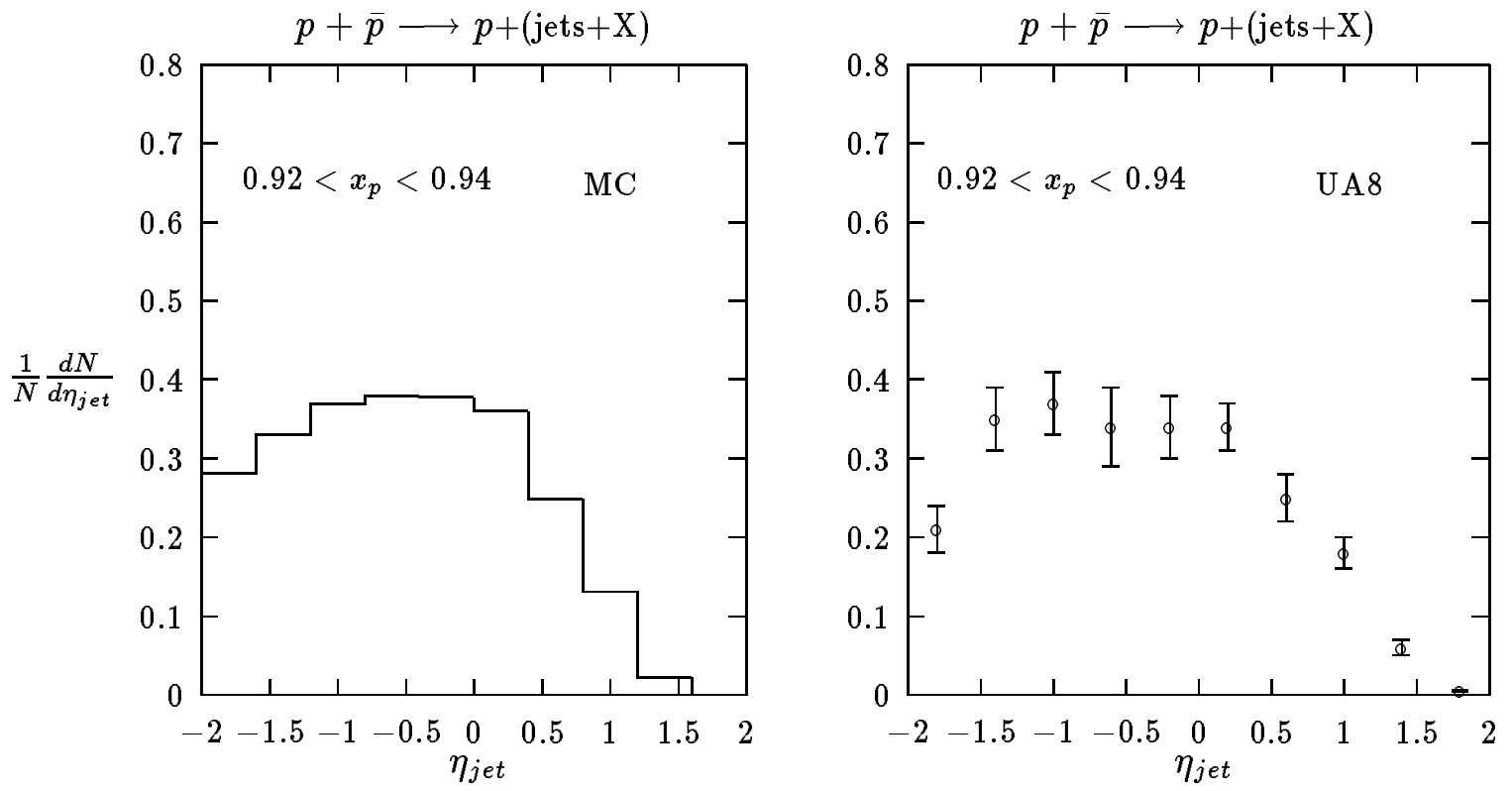

Fig. 12 

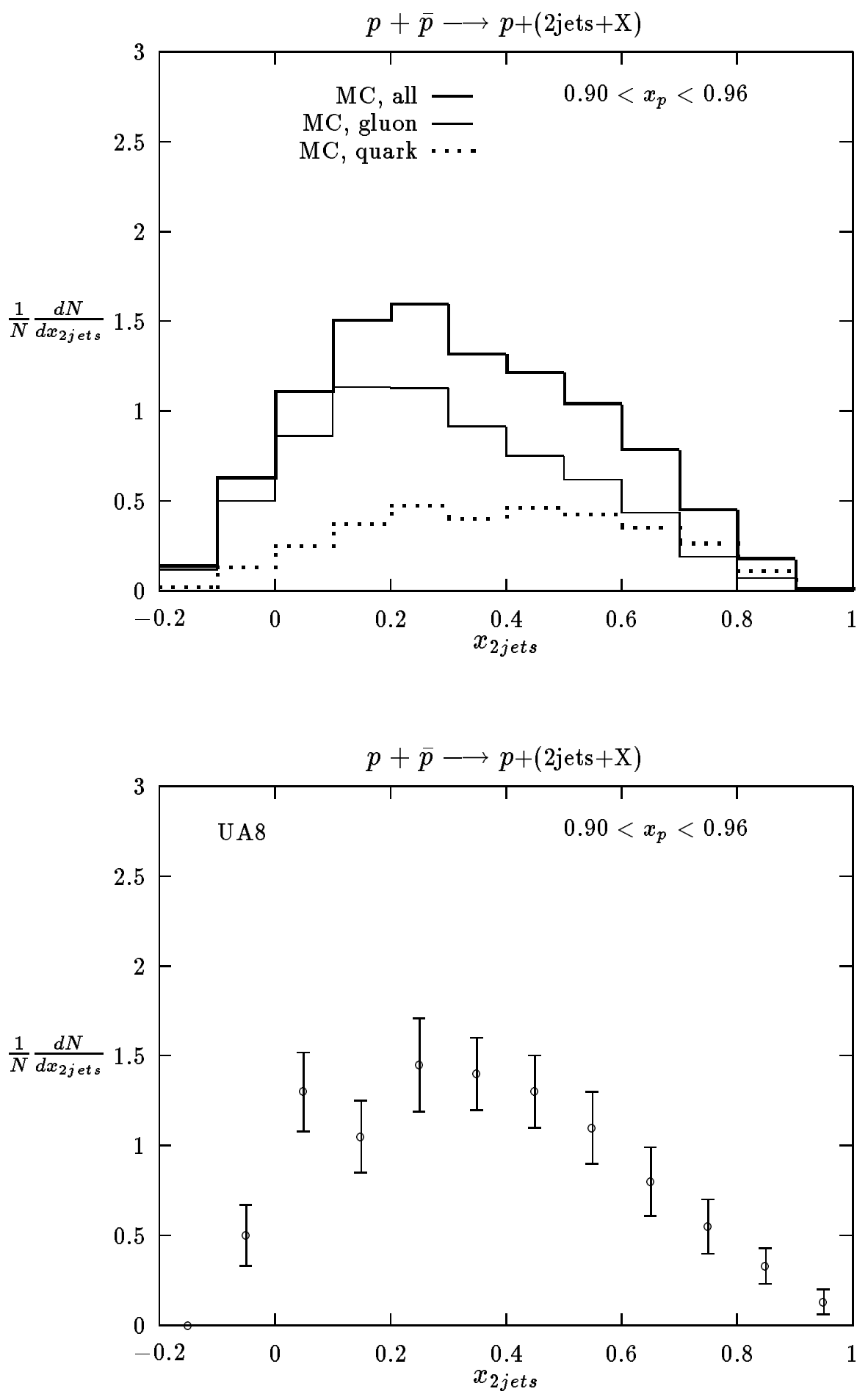

Fig. 13 